\title{
DYNAMICS RESPONSE OF THE HUMAN HEAD DURING DRYWALL IMPACT
}

\author{
Michael A.K. Liebschner ${ }^{a}$ and Leroy R. Waite ${ }^{b}$ \\ ${ }^{a}$ Rimkus Consulting Group, Biomechanics Practice, Houston, Texas \\ ${ }^{\mathrm{b}}$ Rimkus Consulting Group, Biomechanics Practice, Blue Bell, Pennsylvania
}

Corresponding Author: Michael A.K. Liebschner, Ph.D., P.E.,

Rimkus Consulting Group, 12140 Wickchester Lane, Suite 300, Houston, Texas, 77079.

Email: MLiebschner@rimkus.com

https://doi.org/10.34107/BiomedSciInstrum.57.04136

\begin{abstract}
Little experimental data has been reported on the biomechanics of head collisions with drywall sections. The dynamics of head collisions with rigid structures are well documented. However, impacts with compliant, composite structures are more difficult to analyze. The study objective was to correlate the severity of a head impact with damage to the drywall. A human head analog was instrumented with a tri-axial accelerometer and a uniaxial load cell was placed along the cervical spine axis. A randomized block design of drop height and head orientation was utilized. The test results indicated a primarily linear correlation between drop height and peak head acceleration, as well as correlation between drop height and the geometry of the indentation to the drywall. Head posture had little influence on wall damage, however, head extension resulted in a stiffer head-spine complex compared to a flexed posture. A two-factor ANOVA determined a statistically significant correlation between damage severity and impact velocity. The results obtained can be used by accident reconstructionists to approximate the impact severity of a head impacting drywall. The study data are limited to drywall sections of known, similar geometry, and does not apply to scenarios with a support beam directly beneath the drywall. Further studies are needed to investigate additional head postures.
\end{abstract}

Keywords: Dynamic Head Impact, Drywall, Traumatic Brain Injury, Compliant Surface, Wall Impact, Concussion

\section{INTRODUCTION}

The research described in this paper arose because little experimental data has been reported in the scientific literature regarding the dynamics of head collisions with drywall sections. Although head collisions with rigid structures, which are primarily dependent on the impact duration, are well documented [1,2], impacts with compliant, composite structures are more difficult to analyze, therefore suggesting the need for the use of empirical data to assist in the analysis. A suggested working hypothesis for this work, was that an impact to a subject's head that would result in a basilar skull fracture, would cause more severe damage to the drywall section, than what was observed in an exemplar incident. However, such a statement is based on presumptions and not supported by quantitative information. Therefore, the objective of this work was to determine whether the empirical data support the working hypothesis and to correlate the severity of a head impact of a human subject with a drywall bulkheadsection, as quantified by head acceleration and impulse duration, to the dry wall damage.

\section{MATERIALS \& METHODS}

Current reconstruction techniques of head impact employ methodologies which result in measures of primarily time-independent compression forces. However, actual head contacts with a section of a wall typically are dynamic and occur at impact durations that are shorter than a tenth of a second $[3,4]$. 